\title{
Microtiter plate, optode respirometry, and inter-individual variance in metabolic rates among nauplii of Artemia sp.
}

\author{
Tracy L. Szela, Adam G. Marsh* \\ College of Marine Studies, University of Delaware, 700 Pilottown Road, Smith Laboratory, Rm 114, Lewes, \\ Delaware 19958, USA
}

\begin{abstract}
Understanding the potential metabolic lifespan of a cohort of marine invertebrate embryos or larvae requires not just precise measurements of respiration rates, but also requires a large number of individual-level measurements to accurately describe the distribution of metabolic rate potentials within that cohort. To this end, we have developed a simple protocol for converting a 384-well microtiter plate into a 384-chamber, microrespirometer, optode using a plate-reading fluorometer for continuous, real-time data acquisition. We have ground-truthed this high-throughput technique using Artemia sp. nauplii at $\sim 48 \mathrm{~h}$ post-hydration. In this paper we present $>1000$ separate respiration rate measurements on nauplii, providing a novel look at the distribution of metabolic rates within a cohort of larvae. At this high level of individual sampling, we have applied a Shannon-Weaver information entropy statistic to describe the 'complexity' of these rate distributions and to show that the range of metabolic phenotypes expressed in a group of nauplii is responsive to the salinity in which they are rehydrated. Understanding the nature and mechanisms by which variations in metabolic rate intensities can be so large within a cohort and can be responsive to environmental parameters represents a real challenge in larval biology, which will require high-throughput methodologies at both molecular and biochemical levels to decipher.
\end{abstract}

KEY WORDS: Respiration $\cdot$ Optrode $\cdot$ Optode $\cdot$ Artemia sp. $\cdot$ Oxygen $\cdot$ Microtiter plate $\cdot$ Larvae

\section{INTRODUCTION}

Understanding the range of physiological phenotypes in a cohort of embryos or larvae is crucial to understanding the lifespan, dispersal potential, and recruitment success of the early life-history stages of a species. We all tacitly recognize the large biological variation that exists among individual embryos of a cohort, even at the level of maternal investment in individual eggs (McEdward \& Coulter 1987). No propagules are identical. Yet, to date there have only been a few studies of inter-individual variation in metabolic rates of embryos or larvae of marine invertebrates (Marsh et al. 2001a, Peck \& Prothero-Thomas 2002), with most studies of larval lifespan relying on group means of 10 s to 100 s of individuals measured in small volumes.
Although several historical studies have made sensitive measurements of oxygen consumption rates in a few marine invertebrate embryos and larvae (Gray 1925, Spärck 1929, Meyer 1935, Zuethen 1943, Scholander et al. 1952), most of these techniques require too much artisan specialization to utilize, or are too labor-intensive for large numbers of measurements to be made in any single experiment. The oxygen detection methodologies that are now commonly employed require less technical specialization of the operator and can be applied to numerous samples at a time: electrode scanning voltammetry (Glazer et al. 2004), polarographic oxygen sensors (Pamatmat 1997, Marsh \& Manahan 1999, Marsh et al. 2001b), optical fluorescence detection (Marsh et al. 2001a, Gatti et al. 2002, Green 2004), and couloximetry (Peck \& Prothero-Thomas 2002). These methods are all sensi- 
tive enough to detect differences in oxygen concentrations on the order of $2 \mu \mathrm{M}$, ca. $50 \mathrm{pmol} \mathrm{O}_{2}$ in a $25 \mu \mathrm{l}$ volume, and mainly differ in their sample throughput capacity.

Of these techniques, only the optode (Marsh et al. 2001a) and the couloximeter (Peck \& Prothero-Thomas 2002) have been used to measure inter-individual variation in embryonic and larval life stages of marine invertebrates. Both of these studies report substantial differences (by an order of magnitude) in metabolic rates during development within single cohorts. Piecing together the biological significance of why a critical component of physiological function is so variable and understanding the environmental influences that may increase or decrease that metabolic variance requires rate measurements of individuals, but on a 'population' scale. We need to be able to make enough respiration rate measurements to be able to fully describe the population distribution of physiological phenotypes.

In this study, we present a method for measuring individual respiration rates of marine invertebrate embryos and larvae that is sensitive, simple, and has a high throughput. We have incorporated the efficiency of solid-state optical fluorescence detection (optodes: Klimant et al. 1997, 1999) in a 384-well microtiter plate to establish a sensitive 384-chamber respirometer that is unparalleled in its ease of use and high-throughput data acquisition. Using large sample sizes of individual Artemia sp. nauplii, we were able to measure a wide distribution of metabolic rates with variances that are sensitive to changes in environmental salinity.

\section{MATERIALS AND METHODS}

Microtiter plate respirometers. Fluorescence quenching of an organic ruthenium complex [ruthenium(II)tris-4,7-diphenyl-1,10-phenantroline perchlorate] in the presence of oxygen can be directly measured in optode devices (Klimant et al. 1997, 1999, Wang et al. 1999). This quantitative interaction was initially described by Kautsky (1939) as the 'dynamic fluorescence quenching' of oxygen molecules, where the collision between oxygen atoms and an excited fluorophore can result in the non-radiative transfer of energy. In quantitative optodes, the degree of quenching is dependent on collision frequency, which is directly proportional to oxygen concentrations. The primary advantage of optical fluorescence devices to detect oxygen concentrations is that neither the ruthenium fluorophore nor the oxygen molecule are consumed or modified during their interaction, allowing continuous, long-term measurements without removing any oxygen from the system (Krihak \& Shahriari 1996).
We have worked with optodes for several years and have been very impressed with the robust nature of the calibrated response (Marsh et al. 2001a, Glazer et al. 2004). In pursuing applications of this technology to smaller and smaller chamber volumes, we have modified the surface of microtiter plates to function as micro-respiration chambers in a plate-reading fluorometer. We have used both 96- and 384-well formats successfully and, in this work, present only data for the 384-well plates because of the greater technological challenges in working with the smaller wells (40 $\mu \mathrm{l}$ working volume). We used 384-well, black-wall microtiter plates, with glass bottoms (Molecular Machines and Industries). Glass-bottom plates allow fluorescence readings through the bottom of the plate ( $\lambda$ ex: $485 \mathrm{~nm}$; $\lambda$ em: $600 \mathrm{~nm}$ ). They also provide a nonpermeable surface, unlike ordinary microtiter plates that have plastic, gas-permeable membranes on the bottom of the wells. Ruthenium imbedded within thin, fiber-glass films was obtained from OceanOptics. Small, 2-mm diameter discs were cut from the stock sheets using a mouse ear punch (Harvard Apparatus, $2 \mathrm{~mm}$ hole diameter) and were adhered in the center of each well using silicon rubber sealant (Dow Corning/ DAP). Silicon is highly permeable to oxygen; therefore, it does not interfere with diffusion from seawater into the disc (Stokes \& Somero 1999). In order to minimize equilibration periods of the $\mathrm{Ru}^{2+}$ discs, the plates were stored in sterile Milli-Q water to keep them hydrated and then rinsed and equilibrated with filtered seawater (FSW) prior to use. A microtiter plate fluorometer (Flx-800, Bio-Tek Instruments) with 485/20 excitation and 590/35 emission filter sets was used to read the plates using the manufacturer's software (KC4 Ver. 2.7.8).

The Stern-Volmer equation (Eq. 1) was used to standardize the quantitative relationship between ruthenium fluorescence and ambient oxygen concentrations:

$$
\left[\mathrm{O}_{2}\right]=\left[\left(I_{0} / I\right)-1\right] / k
$$

where the oxygen concentration is given as a micromolar value, $I_{0}$ is the fluorescence intensity of anoxic ( $0 \%$ oxygen saturation) seawater, $I$ is the fluorescence intensity of a sample, and $k$ is the Stern-Volmer constant, which is the response ratio of fluorescence between 0 and $100 \%$ oxygen-saturated seawater. Our working range of oxygen concentrations was 200 to $243 \mu \mathrm{M}$, well within the linear calibration range of the Stern-Volmer equation (Krihak \& Shahriari 1996). A 2-point calibration of $0 \%$ and $100 \%$ saturation standards was used for each well (i.e. $2 \mathrm{~mm}$ disc). The Stern-Volmer constant $(k)$ was calculated using the fluorescence of anoxic seawater by loading the sample wells in a glove bag under a nitrogen or argon atmo- 
sphere. The $100 \%$ oxygen-saturation standard used a theoretical concentration calculated from a standard equation (Weiss 1970). Each well had its own unique Stern-Volmer constant due to some inherent microscale-variability in the discs. The Stern-Volmer constants we determined were directly comparable to values reported by the manufacturer. Glove-bag runs were repeated every $2 \mathrm{wk}$ to accurately calibrate the plate. At intervals $>2 \mathrm{wk}$, we could detect a noticeable drift in the $I_{0}$ calibration values.

We have exhaustively examined the diffusion capacity of oxygen through the dense plastic side walls of the plates and mineral oil overlay used to seal the wells. We know that neither is gas-impermeable, but we have extensively evaluated the potential for oxygen diffusion through these mediums and have developed an appropriate methodology for making respiration measurements within a time frame that is far shorter than the diffusion kinetics. To test the gas permeability of the plates, each well in the 384-well microtiter plate was filled with $50 \mu$ l of anoxic FSW (nitrogen purged) inside the oxygen-free environment of a glove bag and covered with approximately $50 \mu \mathrm{l}$ of mineral oil to limit diffusion into the top of the well. The plate was then removed from the glove bag and placed in the fluorometer. Continuous monitoring of the wells over a $4 \mathrm{~h}$ period revealed stable concentrations at $0 \mu \mathrm{M} \mathrm{O}_{2}$ for all wells except for those on the outer edges, where an average increase of $2 \mu \mathrm{M} \mathrm{O}_{2} \mathrm{~h}^{-1}$ over $4 \mathrm{~h}$ was detected (or $\sim 100 \mathrm{pmol} \mathrm{O}_{2} \mathrm{~h}^{-1}$ in $50 \mu \mathrm{l}$ ). However, this is a maximum diffusion rate when the concentration gradient between the wells $10 \%$ saturation) and air is greatest. For experimental measurements, when the oxygen tensions start at the air-saturation value of seawater, the diffusion kinetics become negligible because the concentration of oxygen in the wells is near equilibrium with the room atmosphere. For comparisons we have also tried degassing the mineral oil prior to use, but it does not detectably impact the blank response of an individual well.

Respiration runs were done over a 4 to $6 \mathrm{~h}$ period, recording the fluorescence in each well at a sampling interval of 200 to $250 \mathrm{~s}$ (depending on how quickly the fluorometer could cycle between all the wells loaded on a plate). The total run period was chosen as a time long enough to follow a significant change in oxygen concentration without the organisms experiencing any oxygen stress in the wells $\left(<20 \%\right.$ change in $\mathrm{O}_{2}$ concentration). Over the linear section of the run, the Stern-Volmer equation was used to convert fluorescence values over time to oxygen concentrations. A linear regression on oxygen concentration over time was used to calculate the rate of oxygen consumption in each well. All measurements were made at 21 to $23^{\circ} \mathrm{C}$ (room temperature). A variation of $\pm 2^{\circ} \mathrm{C}$ does not impact the calibration response of the ruthenium matrix (manufacturer's specification: OceanOptics).

Artemia sp. experiments. Artemia sp. cysts (San Francisco Brand) were used in the following experiments. All cysts were hatched at room temperature (21 to $23^{\circ} \mathrm{C}$ ) in FSW supplemented by Instant Ocean brand artificial seawater, when necessary, to reach the desired salinity. Nauplii were sieved from the water, rinsed in $0.22 \mu \mathrm{m}$ FSW, and placed in a clean beaker with FSW. For respiration measurements, the nauplii were transferred in a volume of $40 \mu \mathrm{l}$ into a well on the microtiter plate using a calibrated microliter pipette. For the density-dependent measurements, several individuals were pipetted into a well at a single time. Three different-aged Artemia nauplii were used: 46, 50 , and $68 \mathrm{~h}$ old. The 46 and $50 \mathrm{~h}$ old nauplii were measured as individuals and as multiples in density profiles; the $68 \mathrm{~h}$ old nauplii were measured as individuals. For density profiles, nauplii were counted in the wells after the respiration run with the aid of a dissecting microscope. Control (blank) wells were filled with the same seawater that was used for the animals. Approximately $50 \mu \mathrm{l}$ of mineral oil overlay was added to the top of each well to eliminate oxygen exchange with the atmosphere.

Fluorescence values were recorded for each well over a $6 \mathrm{~h}$ time period at $230 \mathrm{~s}$ intervals, which was the time interval to complete 1 reading across all wells. The fluorescence values were converted to respiration rates as described above. Individual respiration rates were determined after blank corrections by subtracting the average blank rate from the individual well respiration rate. The number of blank wells used per experiment was approximately $10 \%$ of the total number of wells used with animals. If, after blank correction, a sample well had a negative respiration rate (i.e. an increase in oxygen concentration), it was discarded from further processing (generally $<10 \%$ of the observations). We are still trying to further optimize the blank-rate quantification, especially for low respiration rates, but feel that an acceptance rate of $90 \%$ is certainly comparable to the acceptance rate of observations made with other micro-respiration measurements.

Respiration rates of individual Artemia sp. nauplii were measured at 5 different salinities using the microtiter plate respirometers. Artemia sp. cysts were hatched in salinities of $10,20,30,40$, and $50 \%$. A total of 60 individuals per salinity were measured, plus 30 blank wells to maximize use of the microtiter plate space. The respiration run was done on the second day after rehydration (48 $\mathrm{h}$ old nauplii). Individual Artemia sp. were pipetted into the wells with $40 \mu \mathrm{l}$ of FSW at the appropriate salinity. Respiration run parameters were the same as above, but with a $4 \mathrm{~h}$ run 
time and $250 \mathrm{~s}$ intervals. Individual respiration rates were determined after blank corrections by subtracting the average blank rate from the individual well respiration rates.

Statistics. Linear regressions on time-series respiration plots were calculated in Excel (Microsoft Office 2000) after importing the raw fluorescence data from the KC4 analysis software using a Perl script (ActivePerl 5.8, contributed by P. Ulrich). One-way ANOVAs were used for comparing group means, with a Bartlett's test for homogeneity of sample variance, using SPSS (Version 11.0) for comparing groups, with a significance level of $\alpha=0.05$. Data were log- and square-roottransformed because of their skewed distributions, and produced the same results as non-transformed data. The non-transformed data are presented here.

Shannon-Weaver indexes (Eq. 2) were used as quantitative measures of information complexity for individual Artemia respiration rates in the 5 different salinity treatments. They describe the distribution of respiration rates within a treatment group relative to the information complexity of the resulting metabolic rate profile, and, in general, have been found to be of great utility in describing biological distributions (Prus et al. 1999, Shpak \& Churchill 2000, Masifwa et al. 2001, Tam et al. 2001). Thus, they serve as a proxy measure for the diversity of the metabolic rate phenotypes expressed in the salinity treatments. Shannon-Weaver indexes were calculated for each salinity treatment using:

$$
H^{\prime}=-\Sigma p_{i}\left[\log _{(2)}\left(p_{i}\right)\right]
$$

where $H^{\prime}$ is the Shannon-Weaver index and $p_{i}$ is the frequency of the $i$ th respiration class in the sample distribution. A frequency distribution for each sample was produced using 15 bins of unequal span to maximize the discrimination in regions where the frequency distribution changes more rapidly. The same bin classes were applied to each of the 5 salinity sample distributions, essentially to normalize the different numbers of measurements in each treatment population.

\section{RESULTS}

The fluorometer is capable of displaying continuous, real-time plots of data acquisition in each well. We have presented 5 representative, raw data plots from wells with differing numbers of Artemia nauplii in Fig. 1. Because the fluorescence of the ruthenium complex is quenched in the presence of oxygen, an increase in fluorescence indicates a decrease in oxygen concentration. We designate this technique as 'high throughput' because a 4 to $6 \mathrm{~h}$ run with this plate can produce 384 real-time oxygen profiles (raw data as

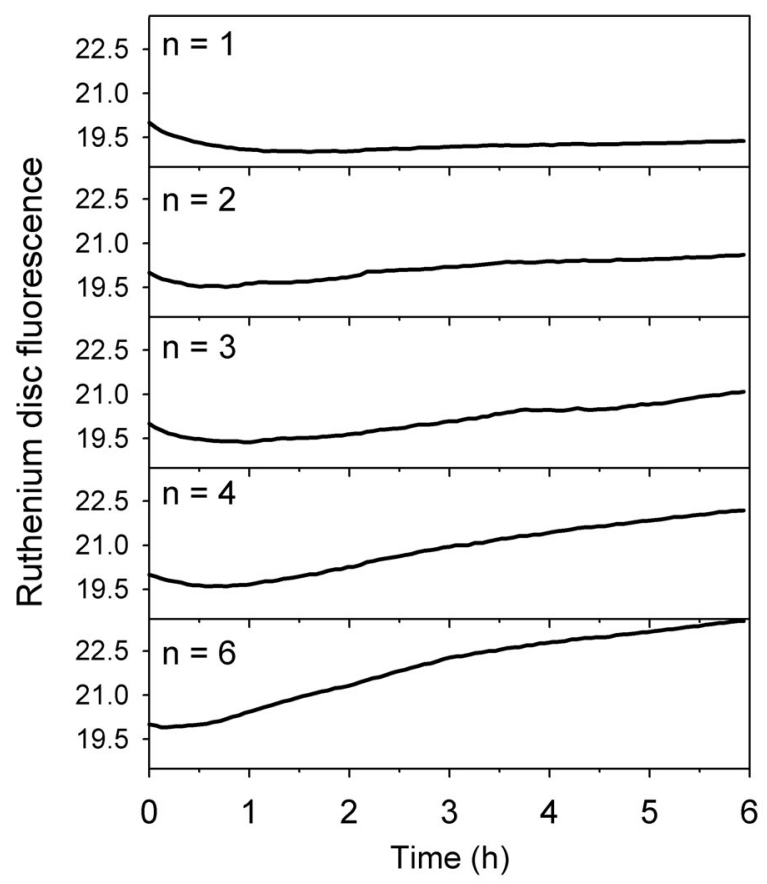

Fig. 1. Artemia sp. Change in fluorescence of 5 selected wells in a 384-well microtiter plate optode with a $40 \mu \mathrm{l}$ assay volume. The presence of oxygen quenches the fluorescence of the $\mathrm{Ru}^{2+}$ fluorophore that coats the bottom of each well, resulting in an increasing fluorescence signal as oxygen is consumed. Each of the wells has a different number of Artemia nauplii indicated by the number at the top left of each panel

in Fig. 1). The primary experimental data presented in this manuscript was generated from 3 plate runs. The volume of data that is output from a run necessitates some computational preprocessing for analysis. Basically, a time interval during which the change in oxygen concentration is linear over time is selected for a regression analysis of $\Delta \mathrm{O}_{2}$ over time. In Fig. 2, a $2 \mathrm{~h}$ interval is shown for 25 selected wells with different numbers of nauplii, with the fluorescence values transformed into oxygen concentrations. These linear regression intervals comprise the processed data sets used to calculate respiration rates. These rates were calculated at the earliest point in time at which the $\Delta \mathrm{O}_{2}$ was constant, and the total oxygen concentration was greater than the $80 \%$ saturation value.

Oxygen consumption rates for 405 individual Artemia nauplii are presented in Fig. 3, subdivided into 3 age classes: 46, 50, and $68 \mathrm{~h}$ post-hydration. The mean respiration rates $( \pm \mathrm{SD})$ for the 3 groups $(112 \pm 57$, $106 \pm 75$, and $113 \pm 90 \mathrm{pmol} \mathrm{O}_{2} \mathrm{~h}^{-1}$, respectively) were not significantly different. The open circles plot observations that lie outside the 10th and 90th percentiles in the distribution and reveal a 30 -fold difference in individual respiration rates within a sample. A secondary approach to estimating individual respiration rates is to 
make measurements of total oxygen consumption with varying numbers of individuals and then to plot the data as a density curve and regress total $\mathrm{O}_{2}$ consumption against total number of individuals (Marsh \& Manahan 1999, Marsh et al. 1999). This calculation is

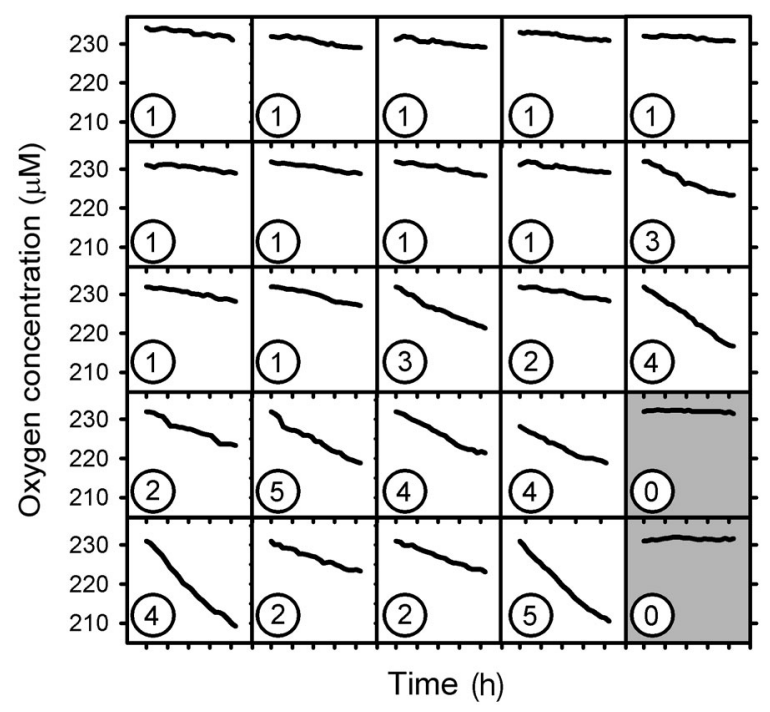

Fig. 2. Artemia sp. Oxygen concentrations in 25 selected wells of a 384-well microtiter plate optode are plotted for a $2 \mathrm{~h}$ time window over which respiration rates were calculated as linear regressions of $\mathrm{O}_{2}$ concentration $(\mu \mathrm{M})$ over time (h). Each tick mark on the time axis represents a 20 min interval. The number of nauplii in a well is indicated at the bottom left of each panel. The gray-shaded panels are blank controls ( $40 \mu \mathrm{l}$ seawater with no animals)

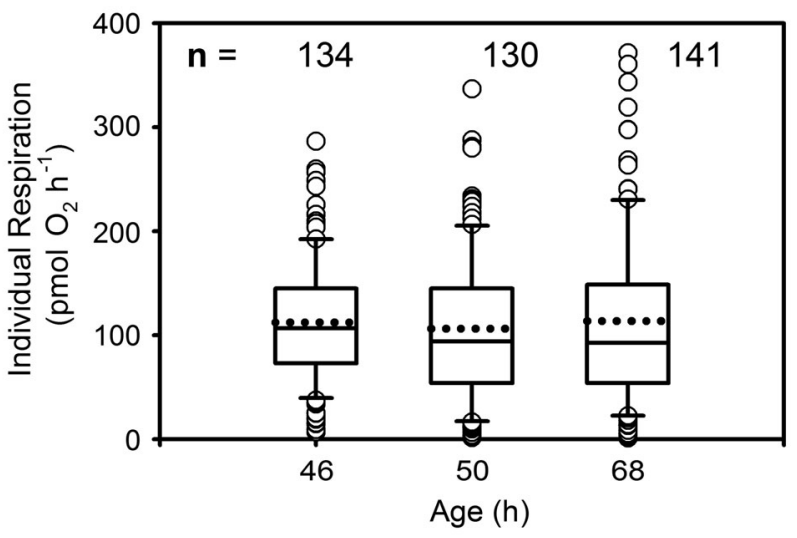

Fig. 3. Artemia sp. Respiration rates of individual nauplii at 3 different ages: 46,50 , and $68 \mathrm{~h}$. The dotted lines represent the means, the solid lines represent the medians, the outer edges of the box represent the 25th and 75th percentiles, and the whiskers represent the 10th and 90th percentiles, with outliers plotted as open circles. Means were 112, 106, and $113 \mathrm{pmol} \mathrm{O}_{2} \mathrm{~h}^{-1}$ and medians were 106, 94, and $92 \mathrm{pmol} \mathrm{O}_{2}$ $\mathrm{h}^{-1}$ for 46,50 , and $68 \mathrm{~h}$ old nauplii, respectively. The number of individual measurements for each age is indicated at the top of the panel performed independently of blank correction, because the regression model partitions the variance in oxygen concentration that is not correlated with number of individuals into a $y$-intercept term. Using this approach with between 1 and 10 nauplii in a well (Fig. 4), the average individual respiration rate was $121 \mathrm{pmol}$ $\mathrm{O}_{2} \mathrm{~h}^{-1}\left(\mathrm{r}^{2}=0.7591, \mathrm{n}=679\right)$. This number is in close agreement with an overall mean value of the individual measurements above $\left(110 \mathrm{pmol} \mathrm{O}_{2} \mathrm{~h}^{-1}\right)$. There is a slight apparent density effect at 10 individuals per well in this plot, which emphasizes why running singleindividual measurements is so important when using small volumes.

To look at the impact of an environmental variable on the distribution of metabolic rates within a treatment group, we hatched Artemia cysts in 5 different salinities: 10, 20, 30, 40, and 50\%. Time to hatching was not noticeably affected by salinity, with nauplii in all treatments appearing by the second day (48 h). Oxygen consumption rates were measured in 320 individuals in 1 plate run (with 40 blanks). Of the total wells set up, 267 yielded linear results in the $2 \mathrm{~h}$ time interval selected for analysis (between the 2 nd and 4 th hour of incubation). After correcting for the blank rates, there were 237 positive respiration rate values (a rejection rate of $12 \%$ ). Of these, 3 were dropped as high-outliers because they were 2-fold greater than the 95th percentile of their respective treatment groups, and wells with dead individuals tend to have high apparent respiration rates most likely because of biochemical oxidation reactions once they expire in a small volume. In Fig. 5, the remaining 234 observations are grouped by salinity treatment, but there were no significant differences in any pairwise comparisons of

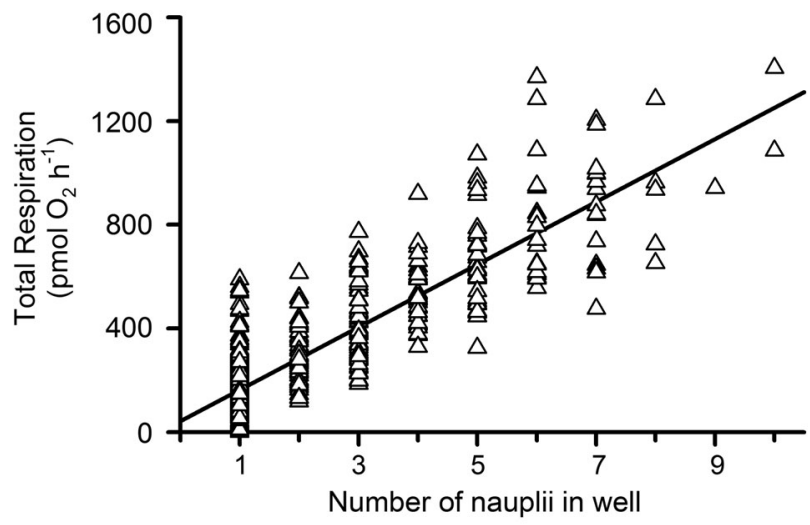

Fig. 4. Artemia sp. Density plot of varying numbers of nauplii per well; 1 to 10 nauplii were used in single wells to calculate an average individual respiration rate. A linear regression of the oxygen concentration against the number of nauplii calculates a slope that represents the average individual respiration rate: $121 \mathrm{pmol} \mathrm{O}_{2} \mathrm{~h}^{-1}$ nauplii-1 $\left(\mathrm{r}^{2}=0.759 ; \mathrm{n}=679\right)$ 


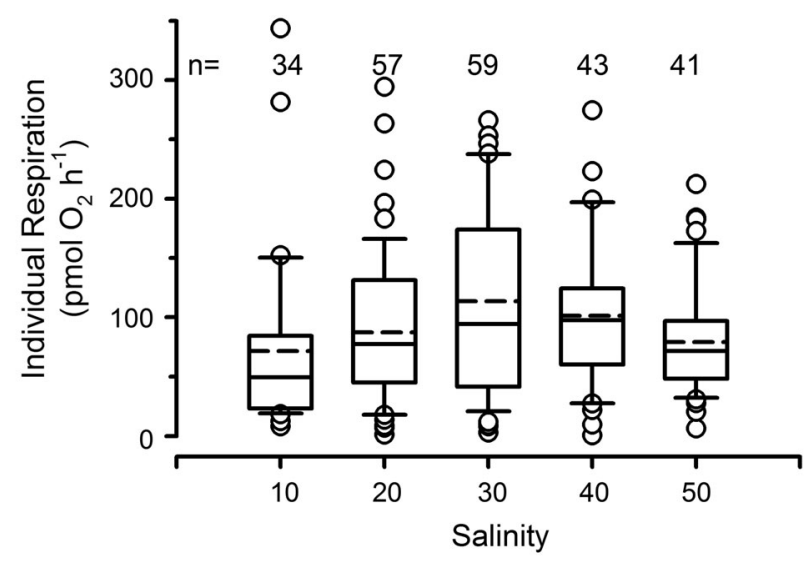

Fig. 5. Artemia sp. The variance in individual respiration rates of $48 \mathrm{~h}$ old nauplii in 5 salinity treatments: 10, 20, 30, 40, and $50 \%$. Box plots are as described in Fig. 3. Group means are $71,87,113,100$, and $78 \mathrm{pmol} \mathrm{O}_{2} \mathrm{~h}^{-1}$, respectively. The number of observations in each group are $34,57,59,43$, and 41, respectively. Even though the observations outside the 10th and 90th percentiles are plotted as outliers, all observations are included in the distribution analyses

the mean respiration rates (Tukey's post hoc test, $\mathrm{p}>>$ 0.05 ; means $=71,87,113,100$, and $78 \mathrm{pmol} \mathrm{O}_{2} \mathrm{~h}^{-1}$, respectively). The rate of $113 \mathrm{pmol} \mathrm{O}_{2} \mathrm{~h}^{-1}$ obtained for the $30 \%$ s treatment is in close agreement with the measurements in Figs. 3 \& 4 that were also made with nauplii cultured at $30 \% \mathrm{~S}$.

Even though the mean metabolic responses in these salinities are not different, there are noticeable changes in the distributions of metabolic rates within a treatment. Because of these large differences, measurements from different plate runs were cross-correlated to see if there was any trend for a well that yielded a high respiration rate in one experiment to also have a high respiration rate in another run. There was no statistical correlation $\left(\mathrm{r}^{2}=0.0217, \mathrm{p}>0.940\right)$ or any trend for a methodological bias in the data based on differences in well responsiveness. The variance observed in individual respiration rates is primarily attributable to biological processes and not methodological artifacts.

In Fig. 6, the respiration rate distributions are plotted to show how, despite having equivalent mean values, these treatment samples are visually different. As a further step towards comparing how these distributions differ, a Shannon-Weaver entropy statistic (information complexity) was used to compare them. Converting the ranked observations (from Fig. 6) into a frequency distribution, we were able to derive a quantitative estimate of the range of potential metabolic rates that exists within a treatment group (Fig. 7). If each individual can be considered to represent a potential metabolic phenotype within a population of

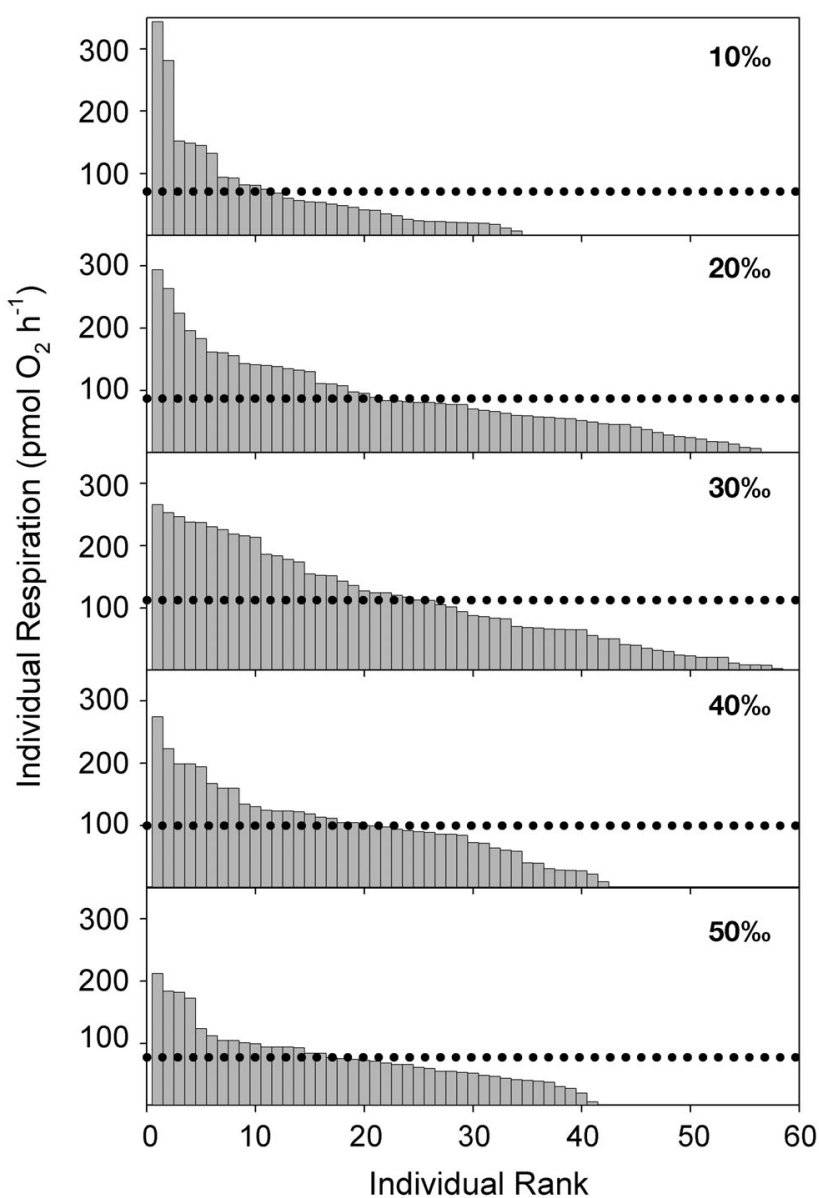

Fig. 6. Artemia sp. Distribution of individual nauplii respiration rates for each of the 5 salinity treatments (as indicated in top right of each panel). Within a treatment, individuals are ranked from highest to lowest. Horizontal dotted lines represent the means (as listed in Fig. 5)

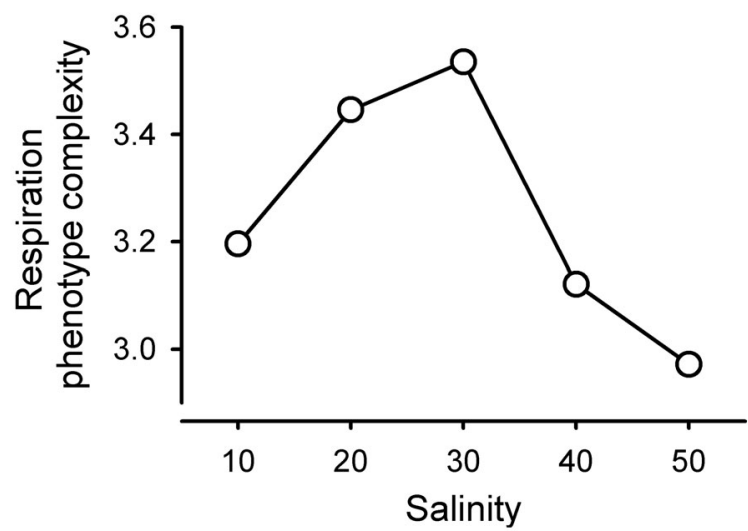

Fig. 7. Artemia sp. A Shannon-Weaver information entropy index describes the relative complexity of the distribution of respiration rates (see Fig. 6) in nauplii raised in different salinities. A frequency distribution of the different respiration rates within a group was obtained using 15 bins or classes for scoring frequency and calculating the probability that an individual nauplii removed from the population would have a respiration rate falling within the $i$ th bin $\left(p_{i}\right.$ in Eq. 2$)$ 
potential phenotypes, then the interpretation of the Shannon-Weaver index is that higher values mean greater phenotypic diversity in a sample group, i.e. a greater information content is expressed in the Artemia nauplii at 10, 20, and $30 \%$ o in comparison to 40 and $50 \%$ S.

\section{DISCUSSION}

\section{Respiration methodologies}

Recently, 2 studies of metabolic rate processes in marine invertebrate embryos and larvae have provided a quantification of inter-individual variances that has not been possible since Scholander's seminal work in the early 1950s using Cartesian divers (Scholander et al. 1952). These recent studies used the userfriendlier methodologies of either optode fiber-optics (Marsh et al. 2001a) or couloximetry (Peck \& ProtheroThomas 2002) to detect changes in oxygen concentrations in the $2 \mu \mathrm{M}$ range. These modern methodologies are not necessarily more sensitive than other techniques, but they are more amenable for working with small volumes and larger sample numbers. The couloximetry method developed by Peck \& ProtheroThomas (2002) has provided some of the lowest, single-individual metabolic rate measurements for any marine embryos (as low as $1 \mathrm{pmol} \mathrm{O}_{2} \mathrm{~h}^{-1}$ in a blastula stage of a polar sea star embryo). These microrespirometers are accurate and can achieve this level of sensitivity by incubating the embryos in sealed capillary tubes for 60 to $70 \mathrm{~h}$. Although sensitive, both of these techniques are ultimately limited by the number of sealed, respiratory chambers that one can physically handle in a single experiment.

The microtiter plate presented here uses optical fluorescence for direct, continuous, real-time detection of oxygen concentrations in several hundred chambers simultaneously. Although the technique has a sensitivity of $\sim 20 \mathrm{pmol} \mathrm{O}_{2} \mathrm{~h}^{-1}$ in the data set presented here, the methodology still warrants further exploration and optimization by other researchers. Two areas currently under consideration to increase the sensitivity are: (1) working out the plate handling routine to include a blank-rate measurement for all individual wells prior to adding embryos or larvae and (2) programming a software package $\left(\mathrm{C}^{++}\right)$to provide greater flexibility and discrimination when running the respiration calculations on such large data sets. Because each of the wells requires a separate calibration calculation, it is likely that in order to make respiration measurements below 20 pmol $\mathrm{O}_{2} \mathrm{~h}^{-1}$ independent blank-rate standardizations for each well will be required. Evaluating the quality of data collected at this scale becomes a time-consuming task when solely relying on a single experimenter to physically analyze the data. Developing a software tool to provide automatic, but intelligent decisions about data parsing and analysis will ensure that the most confident (statistically significant) rate measurements are made for each well. Obviously, the great utility of this technique lies in the number of parallel samples that can be processed, and even a rejection rate of $10 \%$ on a 384 -well plate still yields sample sizes for individual-level rate measurements that are an order of magnitude greater than any previously published studies.

\section{Artemia sp. respiration}

Artemia spp. are found on every continent except Antarctica, inhabiting salt lakes, coastal lagoons, and solar saltworks, regions where the salinity is on average much higher than in the open ocean (Van Stappen 2002). Individual variation of cyst responses to environmental cues has been documented for Artemia spp. by Drinkwater \& Clegg (1991), who recognized the importance of measuring individual metabolic rates of cysts because there may be a continuum of metabolic rates within a cohort. Comparisons of respiration rates between different Artemia spp. studies can be problematic because of differences in the strains of Artemia used. Studies have shown that different strains or even different collection times can produce populations of cysts with known differences in cyst size, chorion thickness, and biomass (Vanhaeckle \& Sorgeloos 1980). Strains also can vary with respect to physiological rate functions and biochemical composition (Dhont \& Sorgeloos 2002). Shilling \& Manahan (1990) show an increase from 352 to $638 \mathrm{pmol} \mathrm{O}_{2} \mathrm{~h}^{-1}$ between 2 to $3 \mathrm{~d}$ old Artemia sp. nauplii. Varo et al. (1993) report a metabolic rate of $65.4 \mathrm{pmol} \mathrm{O}_{2} \mathrm{~h}^{-1}$ for 24 to $28 \mathrm{~h}$ old Artemia sp. nauplii. In general, there is a wide range of metabolic rates reported for Artemia nauplii, and our measurement of $\sim 110 \mathrm{pmol} \mathrm{O}_{2} \mathrm{~h}^{-1}$ at $48 \mathrm{~h}$ fall in the middle of that range.

There are contrasting results for the effect of salinity on respiration rates of Artemia sp. nauplii in the classical literature (Eliassen 1952, Kratowich 1964, Angelovic \& Engel 1968). Recent work has documented a maximum rate at intermediate salinities with a significant impact of acclimation during development (De Wachter \& Van den Abbeele 1991). In our study, mean and median respiration rates were maximal at $30 \%$ S, although not statistically different among salinity treatments. However, with the large individual sample sizes obtained, it is clear that there is a substantial difference in the relative distributions of individual metabolic rates among the treatments (Fig. 6). 


\section{Inter-individual variation in metabolic phenotypes}

Although no one would ever argue that individuals in a population could ever be considered as identical units, applying this perspective to early life stages of marine invertebrates has been limited because of the inherent difficulties in working with very small embryos and larvae on an individual basis. McEdward \& Coulter (1987) measured individual egg size and energy content in sea star embryos and compared these between and among different females. Egg size has traditionally been used in echinoderms as a measure of energy content, but McEdward and Coulter were the first to look at the inter-individual variability and maternal effects of energy content within a cohort. Within 1 spawn there was considerable variation in energy content, and this could not be correlated with egg size (McEdward \& Coulter 1987). The survival or fitness of a cohort is influenced by all the different physiological phenotypes in that cohort. Metabolic rates can be used as a predictor of how long a larva can survive in the water column. For example, a larva with a low metabolic rate can survive longer periods of time without food, because it is not using up the food resources or energy reserves as quickly as a larva with a higher metabolic rate (Bang et al. 2004). Even at the onset of development, differences in metabolic rates can be seen within a cohort of sea urchin zygotes (Scholander et al. 1952).

Work with embryos and larvae of the Pacific oyster Crassostrea gigas has suggested that these animals carry a high genetic load of recessive lethal mutations (Launey \& Hedgecock 2001). The degree of genetic load in an F1 cohort appears to correlate with the variance that can be observed in individual growth performance of early stage larvae. The process of development is a sequential process of gene expression, and, as development proceeds from stage to stage, more and more genes that have previously been silent may be expressed. In organisms with a high genetic load, this can lead to large inter-individual variance in individual phenotypes (morphological and biochemical) and result in the subsequent loss (mortality) of unfit genotypes. In the Pacific oyster, it is possible to detect a significant loss of embryonic genotypes within $6 \mathrm{~h}$ of fertilization (Launey \& Hedgecock 2001).

The most critical aspect of studying inter-individual variation in embryos and larvae is that these life stages present experimentalists with the full range of phenotypes that exist in a cohort. As soon as development begins, the functional performance of those genotypes begins to remove genetic variation from the cohort. Even at the level of gene-expression profiles, large differences among larvae can be observed (Marsh \& Fielman 2005); this substantiates the conclusion that all larvae in a co- hort are not created equal. In unpredictable environments, it is likely to be adaptive to produce such high levels of variance within a cohort so that at least some individual larvae will encounter conditions that are optimal for their genotype performance.

The 30-fold difference in metabolic rates found in this study may suggest that many of those highmetabolism phenotypes are not likely to survive to the end of the larval development period. Previous work with another crustacean nauplii (Marsh et al. 2001a) has described a 3-fold variation in oxygen consumption rates, but this measurement was obtained with a sample size of just 10 individuals per respiration run. The impact of increasing the sample size in this study to $>1000$ individuals increases the probability of sampling outliers in the distribution of metabolic phenotypes, providing a broader assessment of phenotypic variability.

In the present study, we see individual rates varying by as much as 30 -fold within the salinity treatments. We have used a Shannon-Weaver entropy index to describe the distribution of metabolic states within each treatment population (Fig. 7). The number of physiological phenotypes showed the largest shift between 30 and $40 \%$ S, with both the 40 and $50 \%$ S treatments evidencing a $\sim 15 \%$ reduction in information complexity. Understanding the nature and mechanisms by which variations in metabolic rate intensities can be so large within a cohort and can be responsive to environmental parameters represents a real challenge in larval biology. Pursuing these questions of variance requires experimentation with high throughput to adequately sample sufficient numbers of individuals to construct a population-level context. Such methodologies of high through-put can place larval biology at the frontier of environmental genomics aimed at cross-mapping the determinants of interindividual variation in rates of gene expression, biochemical function, and physiological activity.

Acknowledgements. We thank G. Luther III for assistance with the anaerobic work, D. Miller for statistical consultation, J. Garcia-Frias for discussions on information entropy, D. Stokes for invaluable advice in the construction of optodes, and P. Ulrich for a PERL script. This work was supported by a grant from the National Science Foundation program in Biological Oceanography (OCE-0095459).

\section{LITERATURE CITED}

Angelovic JW, Engel DW (1968) Interaction of gamma irraditation and salinity on respiration of brine shrimp (Artemia salina) nauplii. Radiation Res 35:102-108

Bang A, Gronkjaer P, Malte H (2004) Individual variation in the rate of oxygen consumption by zebrafish embryos. J Fish Biol 64:1285-1296

De Wachter B, Van den Abbeele J (1991) The influence of 
acclimation on salinity and oxygen on the respiration of the brine shrimp, Artemia franciscana. Comp Biochem Physiol A 98:293-298

Dhont J, Sorgeloos P (2002) Applications of Artemia. In: Abatzopoulous TJ, Beardmore JA, Clegg JS, Sorgeloos P (eds) Artemia: basic and applied biology, Vol 1. Kluwer Academic Publishers, Boston, MA

Drinkwater LE, Clegg JS (1991) Experimental biology of cyst diapause. In: Browne RA, Sorgeloos P, Trotman CNA (eds) Artemia biology. CRC Press, Boca Raton, FL, p 374-396

Eliassen E (1952) The energy-metabolism of Artemia salina in relation to body size, seasonal rhythms, and different salinities. Univ Bergen Arbok Naturvit Rekke 11:1-17

Gatti S, Brey T, Muller WEG, Heilmayer O, Holst G (2002) Oxygen microoptodes: a new tool for oxygen measurements in aquatic animal ecology. Mar Biol 140:1075-1085

Glazer BT, Marsh AG, Stierhoff K, Luther GW III (2004) The dynamic response of optical oxygen sensors and voltammetric electrodes to temporal changes in dissolved oxygen concentrations. Anal Chim Acta 518:93-100

Gray J (1925) The mechanism of cell division. II. Oxygen consumption during cleavage. Biol Rev 1:227-236

Green BS (2004) Embryogenesis and oxygen consumption in benthic egg clutches of a tropical clownfish, Amphiprion melanopus (Pomacentridae). Comp Biochem Physiol A 138:33-38

Kautsky H (1939) Quenching of luminescence by oxygen. Trans Faraday Soc 35:216-219

Klimant I, Kuhl M, Glud RN, Holst G (1997) Optical measurement of oxygen and temperature in microscale: strategies and biological applications. Sens Actuat B 38:29-37

Klimant I, Ruckruh F, Liebsch G, Stangelmayer C, Wolfbeis OS (1999) Fast response oxygen micro-optodes based on novel soluble ormosil glasses. Mikrochim Acta 131:35-46

Kratowich NR (1964) The effect of metabolic inhibitors on osmoregulation by nauplii of Artemia salina at various salinities. Am Zool 4:389-390

Krihak M, Shahriari MR (1996) A highly sensitive, all solid state fiber optic oxygen sensor based on the sol-gel coating technique. Electron Lett 32

Launey S, Hedgecock D (2001) High genetic load in the Pacific oyster Crassostrea gigas. Genetics 159:255-265

Marsh AG, Fielman KT (2005) Transcriptome profiling of individual larvae of two different developmental modes in the poecilogonous polychaete Streblospio benedicti (Spionidae). J Exp Zool Mol Dev Evol 304B:238-249

Marsh AG, Manahan DT (1999) A method for accurate measurements of the respiration rates of marine invertebrate embryos and larvae. Mar Ecol Prog Ser 184:1-10

Marsh AG, Leong PKK, Manahan DT (1999) Energy metabolism during embryonic development and larval growth of an antarctic sea urchin. J Exp Biol 202:2041-2050

Marsh AG, Cohen S, Epifanio CE (2001a) Larval energy metabolism and physiological variability in the Asian shore crab Hemigraspus sanguineus. Mar Ecol Prog Ser 218:303-309

Marsh AG, Maxson RE, Manahan DT (2001b) High macromolecular synthesis with low metabolic cost in antarctic sea urchin embryos. Science 291:1950-1952

Masifwa WF, Twongo T, Denny P (2001) The impact of water hyacinth, Eichhornia crassipes (Mart) Solms on the abundance and diversity of aquatic macroinvertebrates along

Editorial responsibility: Otto Kinne (Editor-in-Chief), Oldendorf/Luhe, Germany the shores of northern Lake Victoria, Uganda. Hydrobiologia 452:79-88

McEdward LR, Coulter LK (1987) Egg volume and energetic content are not correlated among sibling offspring of starfish: implications for life-history theory. Evolution 41: 914-917

Meyer H (1935) Die Atmung von Asterias rubens und ihre Abhängigkeit von verschiedenen Außenfaktoren. Zool Jahrb Abt Allg Zool Physiol Tiere 55:349-398

Pamatmat MM (1997) Non-photosynthetic oxygen production and non-respiratory oxygen uptake in the dark: a theory of oxygen dynamics in plankton communities. Mar Biol 129:735-746

Peck LS, Prothero-Thomas E (2002) Temperature effects on the metabolism of larvae of the Antarctic starfish Odontaster validus, using a novel micro-respirometry method. Mar Biol 141:271-276

Prus T, Prus M, Bijok P (1999) Diversity of invertebrate fauna in littoral of shallow Myczkowce dam reservoir in comparison with a deep Solina dam reservoir. Hydrobiologia 409: 203-210

Scholander PF, Claff CL, Sveinsson SL, Scholander SI (1952) Respiratory studies of single cells. III. Oxygen consumption during cell division. Biol Bull (Woods Hole) 102: 185-199

Shilling FM, Manahan DT (1990) Energetics of early development for the sea urchins Strongylocentrotus purpuratus and Lytechinus pictus and the crustacean Artemia sp. Mar Biol 106:119-127

Shpak M, Churchill GA (2000) The information content of a character under a Markov model of evolution. Mol Phylogenet Evol 17:231-243

Spärck R (1929) Studies on the biology of the oyster (Ostrea edulis). VI-VIII. Rep Dan Biol Stn 35:99-110

Stokes MD, Somero GN (1999) An optical oxygen sensor and reaction vessel for high-pressure applications. Limnol Oceanogr 44:189-195

Tam L, Derry AM, Kevan PG, Trevors JT (2001) Functional diversity and community structure of microorganisms in rhizosphere and non-rhizosphere Canadian arctic soils. Biodivers Conserv 10:1933-1947

Vanhaeckle P, Sorgeloos P (1980) International study on Artemia. IV. The biometrics of Artemia strains from different geographical origins. In: Persoone $\mathrm{G}$, Sorgeloos $\mathrm{P}$, Roels O, Jaspers E (eds) The brine shrimp Artemia, Vol 3. Universa Press, Wetteren, p 456-458

Van Stappen G (2002) Zoogeography. In: Abatzopoulous TJ, Beardmore JA, Clegg JS, Sorgeloos P (eds) Artemia: basic and applied biology, Vol 1. Kluwer Academic Publishers, Boston, MA, p 171-224

Varo I, Taylor AC, Amat F (1993) Comparative study of the effects of temperature, salinity and oxygen tension on the rates of oxygen consumption of nauplii of different strains of Artemia. Mar Biol 117:623-628

Wang W, Reimers CE, Wainright SC, Shahriari MR, Morris MJ (1999) Applying fiber-optic sensors for monitoring dissolved oxygen. Sea Technol 40:69-74

Weiss RF (1970) The solubility of nitrogen, oxygen and argon in water and seawater. Deep-Sea Res 20:291-303

Zuethen E (1943) A Cartesian Diver micro respirometer with a gas volume of $0.1 \mathrm{cl}$. CR Trav Lab Carlsberg Ser Chim 24:479-518

Submitted: August 3, 2004; Accepted: March 8, 2005

Proofs received from author(s): June 17, 2005 